\title{
Constraints in the use of repair half times and mathematical modelling for the clinical application of HDR and PDR treatment schedules as an alternative for LDR brachytherapy
}

\author{
L.A.M. Pop*a ${ }^{*}$ J.F.C.M. van den Broek ${ }^{a}$, A.G. Visser ${ }^{b}$, A.J. van der Kogel ${ }^{a}$ \\ ${ }^{a}$ Institute of Radiotherapy, University of Nijmegen, PO. Box 9101, $5500 \mathrm{HB}$ Nijmegen, The Netherlands \\ ${ }^{\mathrm{b}}$ Dr. Daniel den Hoed Cancer Center/Erasmus University Rotterdam, Rotterdam, The Netherlands
}

Received 13 April 1995; revision received 15 November 1995; accepted 7 December 1995

\begin{abstract}
Using theoretical models based on radiobiological principles for the design of new treatment schedules for HDR and PDR brachytherapy, it is important to realise the impact of assumptions regarding the kinetics of repair. Extrapolations based on longer repair half times in a continuous LDR reference scheme may lead to the calculation of dangerously high doses for alternative HDR and PDR treatment schedules. We used the clinical experience obtained with conventional ERT and LDR brachytherapy in head and neck cancer as a clinical guideline to check the impact of the radiobiological parameters used. Biologically equivalent dose (BED) values for the in clinical practice of LDR brachytherapy recommended dose of $65-70 \mathrm{~Gy}$ (prescribed at a dose rate between $30-50 \mathrm{cGy} / \mathrm{h}$ ) are calculated as a function of the repair half time. These BED values are compared with the biological effect of a clinical reference dose of conventional ERT with $2 \mathrm{~Gy} /$ day and complete repair between the fractions. From this comparison of LDR and ERT treatment schedules, a range of values for the repair half times of acute or late responding tissues is demarcated with a reasonable fit to the clinical data. For the acute effects (or tumor control) the best fits are obtained for repair half times of about $0.5 \mathrm{~h}$, while for late effects the repair half times are at least $1 \mathrm{~h}$ and can be as high as $3 \mathrm{~h}$. Within these ranges of repair half times for acute and late effects, the outcome of 'alternative' HDR or PDR treatment schedules are discussed. It is predominantly the late reacting normal tissue with the longer repair half time for which problems will be encountered and no or only marginal gain is to be expected of decreasing the dose rate per pulse in PDR brachytherapy.
\end{abstract}

Keywords: Brachytherapy; Dose rate; Radiobiology; Kinetics of repair

\section{Introduction}

In the design of new fractionation schedules for high dose rate (HDR) and/or pulsed dose rate (PDR) brachytherapy as compared to low dose rate (LDR) brachytherapy $[4-6,13,16]$, it is important to realise the impact of the assumed values of the repair factors in the calculations of isoeffective doses. Experimental and clinical data show a reasonable consistency in the repair capacity ( $\alpha / \beta$ ratio) for both the acute (tumor) and late responding tissues under the conditions of complete repair. However, so far no clear picture has emerged about the kinetics of repair $\left(t_{1 / 2}\right)$ for the conditions of

\footnotetext{
* Corresponding author.
}

incomplete repair during continuous irradiation. Using generalised values for the kinetics of repair [13] or values derived from hyperfractionated external beam irradiation [6] or cells in culture [4], it is important to check the outcome of the predicted isoeffective dose with the clinical experience already obtained. Extrapolations based on longer repair half times in a continuous LDR reference scheme may lead to the calculation of unrealistic and dangerously high doses for alternative HDR and PDR treatment schedules. Moreover, clinical logistics, patient convenience and physical aspects of the rapid dose fall off during brachytherapy need also be taken into consideration.

The purpose of this paper is not to introduce a new radiobiological model, but to discuss consequences in 
clinical practice of predicting isoeffective doses of different treatment schedules for HDR, PDR and LDR brachytherapy. We will demonstrate the pitfalls of extending the BED concept to multifractionated HDR/ PDR brachytherapy, specifically with respect to:

- the choice of the reference treatment,

- the impact of the assumption of the value for the repair half time,

- the application of model parameters in situations quite different from those in which they were derived.

Based on the clinical experience already obtained with LDR brachytherapy (dose rate $<1 \mathrm{~Gy} / \mathrm{h}$ ) in head and neck cancer and a clinical reference dose of conventional external beam irradiation (ERT, 2 Gy per fraction, 5 times per week), a range of values for the repair half times and isoeffective doses is demarcated which show a reasonable fit of the clinical data. Within these ranges of repair half times and BED values for the acute and late effects, the feasibility of 'alternative' HDR (at a dose rate of $120 \mathrm{~Gy} / \mathrm{h}$ ) or PDR (dose rate $1-3 \mathrm{~Gy} / \mathrm{h}$ ) treatment schedules is discussed, where practical considerations in the clinical use of brachytherapy are taken into account; especially with regard to the possibility of disconnecting the patient from the afterloading HDR or PDR machine during the night and/or between the pulses.

\section{Methods}

\subsection{Clinical reference doses of $L D R$ brachytherapy in} head and neck cancer as compared to conventional external beam radiation therapy

If LDR brachytherapy is used as a single modality in treating cancer of the oral cavity, the recommended dose is $65-70$ Gy prescribed at a reference dose rate between $30-50 \mathrm{cGy} / \mathrm{h}[23,27]$, with as a consequence an overall treatment time limited to about 1 week. For such schedules an increase of local tumor control as compared with conventional ERT alone or conventional ERT combined with an interstitial implant was observed, with an acceptable number of (late) normal tissue complications [3,7,18,22-24]. However, due to variations in dose distribution, tumor size and irradiated volume, a straightforward comparison of LDR brachytherapy and conventional ERT is not possible. Brachytherapy is usually preferred in treating small tumor sizes. For any given dose of radiation, tumor control as well as normal tissue tolerance will increase with smaller volumes. Moreover, the physical advantage of the rapid dose fall off around the implanted radioactive sources enables high(er) doses to be delivered in the tumor, with relative sparing of the surrounding normal tissues. In the different 'systems' providing rules for the source geometry and dose specification, like the Manchester or Paris system, the mean central dose in the target volume is about $10-15 \%$ higher than the prescribed (reference) dose at the tumor periphery $[12,25]$. This means that if a total dose of 70 Gy LDR brachytherapy is prescribed at a reference dose rate of $50 \mathrm{cGy} /$ hour, the total dose (at a higher dose rate) in the center of the target volume will be at least $77 \mathrm{~Gy}$. This is in contrast with ERT, where the whole (homogeneously) irradiated volume receives the same total dose. As a consequence the total dose which can be applied to the tumor is limited by the tolerance dose of the surrounding normal tissues included in the irradiation field. Taking these considerations into account one could imagine that from the clinical point of view the same biological effect in terms of tumor control and (late) normal tissue complications obtained with 65-77 Gy LDR brachytherapy at a dose rate between $30-55 \mathrm{cGy} / \mathrm{h}(=$ recommended dose with an uncertainty in dose and dose rate of $+10 \%$ ) can be duplicated if it were possible to deliver at least the same total dose of conventional ERT (= 64-76 Gy, if 2 Gy per fraction is used). The clinical experience of Wang et al. [38] with intra-oral cone (external beam) irradiation for selected carcinomas of the oral cavity can be used as a justification for the adoption of this range of total doses conventional ERT between 64 and $76 \mathrm{~Gy}$. Small intra-oral lesions were irradiated with a relatively homogeneous external beam of $280 \mathrm{kV} \mathrm{X}$ rays or 9-12 MeV electrons alone or in combination with external beam Cobalt ${ }^{60}$ irradiation. With this technique it was possible to deliver high doses up to approximately 80 Gy (recalculated by Wang for 2 Gy per fraction each day, 5 days per week) to the primary lesion and to obtain the same level of local tumor control without an increase in late normal tissue complications as compared to LDR interstitial brachytherapy.

\section{2. (In) complete repair model and BED concept (see also appendix)}

Several authors $[2,8,9,17,19,26,31]$ have shown that the LQ model and the ERD or BED concept can be applied to any type of treatment; not only to fractionated ERT with complete repair, but also to conditions of incomplete repair. If fractions are spaced sufficiently to ensure complete repair, the biological effect of a conventional (HDR) fractionation scheme of $n$ fractions of $d$ Gy each is

$B E D_{E R T}=n d\left(1+\frac{d}{\alpha / \beta}\right)-\frac{\gamma \cdot T}{\alpha}$

where $\gamma \cdot T / \alpha$ is a term to correct for the loss in biological effect due to repopulation of rapidly proliferating cells in tumors and early reacting normal tissues $[2,10,33,34]$. 
$T$ is the time during which proliferation occurs at the (assumed fixed) rate $\gamma$, after any initial time lag. The factor $\gamma$ can be replaced by $\gamma=\ln 2 / T_{\text {pot }}$, where $T_{\text {pot }}$ is the potential doubling time of the proliferating cells (assuming the cell loss factor $=0$ ). In general for late effects the time correction factor for repopulation is omitted [15], although for some late reacting normal tissues an indication for the existence of a time factor has been found (for review see ref. [33]).

For continuous LDR irradiation a dose rate dependency has been incorporated in the LQ formalism to correct for incomplete repair during the irradiation $[8,17,31]$, where

$\mathrm{BED}_{\mathrm{LDR}}=d \cdot\left(1+\frac{g \cdot d}{(\alpha / \beta)}\right)-\frac{\gamma \cdot T}{\alpha}$

This function $g$ depends on the total irradiation time $(T)$ and the kinetics of repair represented by the repair half time $\left(t_{1 / 2}\right)$. Assuming that the rate of repair is a monoexponentional function of time and dose (rate) independent:

$g=\frac{2}{\mu \cdot T}\left(1-\frac{1}{\mu \cdot T} \cdot\left[1-e^{-\mu T}\right]\right)$

where $\mu=\frac{\ln 2}{t_{1 / 2}}$

When we invoke the LQ and BED concept for isoeffective dose calculations for the acute (tumor control) and late effects, the BED value for conventional ERT under the condition of complete repair must be equal to the BED value for LDR brachytherapy under the condition of incomplete repair, thus $\mathrm{BED}_{\mathrm{ERT}}$ (acute) = $\mathrm{BED}_{\mathrm{LDR}}$ (acute) or $\mathrm{BED}_{\mathrm{ERT}}$ (late) $=\mathrm{BED}_{\mathrm{LDR}}$ (late)

\subsection{Radiobiological parameters based on clinical data}

In order to characterise the two main response categories (acute vs. late effects) under conditions of complete repair, we will use in our calculations an $\alpha / \beta$ ratio of 10 for the acute reacting normal tissues and tumors and $\alpha / \beta=3$ for the late responding tissues, which are generally accepted values characterising the fractionation sensitivity of the different types of tissues $[15,33]$. Due to uncertainties about $T_{\text {pot }}$, individual knowledge of $\alpha$ and delay before onset of proliferation, it is difficult to define the correction factor for repopulation in the acute reacting normal tissues and tumors. We will use a correction factor for repopulation as originally proposed by Withers in 1988 [39]. Based on an analysis of a large body of clinical data Withers estimated the rate of tumor regrowth during ERT in head and neck cancer. He concluded that with an extension of the treatment time beyond 28 days, about 0.6 Gy per day is lost due to the negative effect of accelerated proliferation on tumor control. In our calculations of the BED for a con- ventional ERT irradiation scheme of $2 \mathrm{~Gy} /$ day, 5 times per week., we will use $0.55 \mathrm{~Gy} /$ day loss after day 28 to correct for repopulation. For a total dose of $70 \mathrm{~Gy}$ in 7 weeks, 18 days remain after a period of 28 days, which means that $0.55 \times 18=9.9 \mathrm{~Gy}$ is effectively lost in compensating for concurrent repopulation. This time correction factor may be an underestimation for very fast proliferating tumors $[10,14,15]$. With regard to the time of onset and rate of the accelerated repopulation after the start of irradiation, there is still much debate about the mechanism and consequences for clinical practice. However, the original time factor of about $0.6 \mathrm{~Gy} / \mathrm{day}$ after a time lag period of about 4 weeks as proposed by Withers, has been confirmed in a number of subsequent analyses of clinical data in head and neck cancer $[20,28,30]$. Because the total irradiation time to deliver 70 Gy L.DR is limited to about 1 week we assume that no cell proliferation occurs during this short treatment time and therefore the second term in the calculation of the BED) value for the LDR irradiation is omitted. For very fast proliferating cells this may be an underestimation, as has been calculated by Dale for the theoretical situation that the potential doubling time of tumor cells is $<3.5$ days [10].

\section{Results}

\subsection{Isoeffective dose calculations of the clinical reference doses of LDR brachytherapy and conventional ERT}

In Fig. 1 the biological effect in terms of BED for the in clinical practice recommended dose and dose rate of LDR brachytherapy is calculated as a function of the repair half time (for the acute effects in Fig. la the BED is expressed in $\mathrm{Gy}_{10}$ assuming $\alpha / \beta=10$, for the late effects in Fig. 1b the BED is expressed in $\mathrm{Gy}_{3}$ assuming an $\alpha / \beta=3$ ). The recommendation of a dose of $65-70 \mathrm{~Gy}$ and a reference dose rate between $30-50 \mathrm{cGy} / \mathrm{h}$ to $\max$ imize local tumor control and minimize late normal tissue effects, includes a dose and dose rate uncertainty between a minimum of $65 \mathrm{~Gy}$ at a dose rate of $30 \mathrm{cGy} / \mathrm{h}$ and a maximum of $77 \mathrm{~Gy}$ at $55 \mathrm{cGy} / \mathrm{h}$. The calculated BED values for these minimum and maximum LDR irradiation schemes are compared with a clinical reference dose of conventional ERT at $2 \mathrm{~Gy} /$ day on the right Yaxis (with a correction in Fig. 1a for tumor repopulation). The BED lines of $70 \mathrm{~Gy} \mathrm{LDR}$ at $50 \mathrm{cGy} / \mathrm{h}(=140$ h) and the $70 \mathrm{~Gy}$ conventional ERT serve as reference lines.

As can be seen in Fig. 1a, a match between clinically equivalent schedules for the acute effects (= tumor control and acute reacting normal tissues) either given as 70 Gy LDR at a dose rate of $50 \mathrm{cGy} / \mathrm{h}$ or $70 \mathrm{~Gy}$ ERT, can only be obtained at a repair half time of $0.2 \mathrm{~h}$. Based on clinical experience of LDR brachytherapy and conventional ERT in head and neck cancer it has been argued 

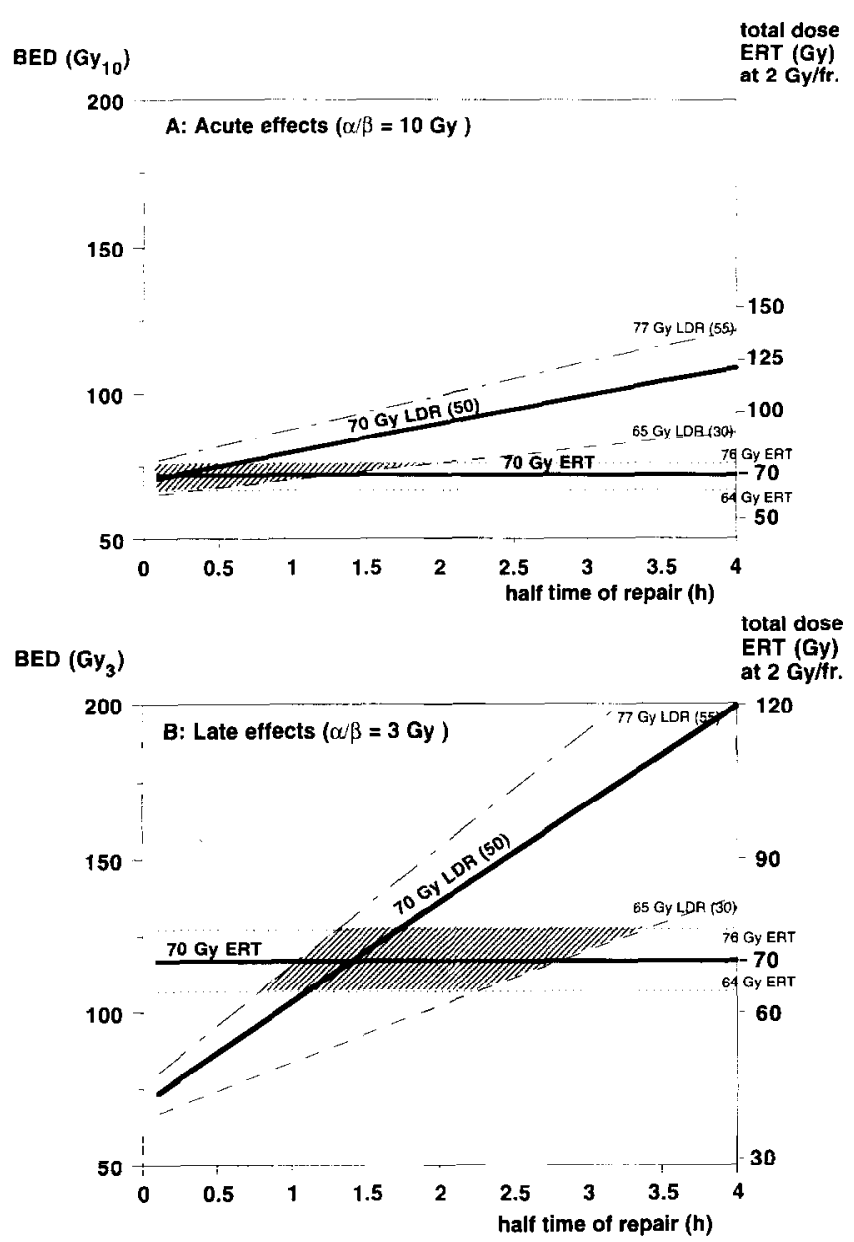

Fig. 1. (A) BED in $\mathrm{Gy}_{10}$ as a function of the repair half time for the acute effects (= tumor control) with an $\alpha / \beta$ ratio of $10 \mathrm{~Gy}$. The calculated BED values on the left $Y$-axis are compared with a biologically equivalent total dose conventional ERT at 2 Gy/day (corrected for repopulation) on the right $\mathrm{Y}$-axis. The oblique lines represent the reference schemes of the in clinical practice recommended dose of 65 or 70 Gy LDR brachytherapy, prescribed at a dose rate between 30-50 cGy/h, with an uncertainty in dose and dose rate between a maximum of $77 \mathrm{~Gy}$ at $55 \mathrm{cGy} / \mathrm{h}$ and a minimum of $65 \mathrm{~Gy}$ at 30 cGy/h. The horizontal lines are the BED lines of 64,70 and $76 \mathrm{~Gy}$ conventional ERT. The shaded area demarcates the range of repair half times and BED values where the LDR and ERT treatment schedules match for tumor control (= acute effects). (B) BED in $\mathrm{Gy}_{3}$ as a function of the repair half time for the late effects with an $\alpha / \beta$ ratio of 3 Gy. Otherwise the same as in (A), with the exception that on the right $\mathbf{Y}$-axis no correction for repopulation has been made. The shaded area demarcates the range of repair half times and BED values where the LDR and ERT treatment schedules match for late effects.

(see previous section) that it is precarious to attribute clinical equivalency of tumor control obtained with 65 or $70 \mathrm{~Gy} \mathrm{LDR} \mathrm{to} \mathrm{one} \mathrm{absolute} \mathrm{value} \mathrm{of} 70 \mathrm{~Gy}$ ERT. Therefore, a range of total doses ERT between 64 and $76 \mathrm{~Gy}$ was proposed (see Methods) to include an uncertainty in total dose of conventional ERT which could result in the same probability of local tumor control as obtained with 65-70 Gy LDR brachytherapy prescribed at a dose rate between $30-50 \mathrm{cGy} / \mathrm{h}$. The shaded area in Fig. 1a. demarcates values of the intrinsic repair half times and BED values for tumor control (= acute effects) to match tumor control obtained within a range of total doses for conventional ERT between 64-76 Gy and the minimum of $65 \mathrm{~Gy}$ at $30 \mathrm{cGy} / \mathrm{h}$ or the maximum of $77 \mathrm{~Gy}$ at $55 \mathrm{cGy} / \mathrm{h}$ as recommended in clinical practice of LDR brachytherapy. Within the boundaries of this shaded area, the repair half time with the highest probability varies between $0.2-1 \mathrm{~h}$. This is in agreement with most experimental data on acute effects. Moreover, in this way it can be easily seen that for tumors or the acute reacting normal tissues, a repair half time $>1.5 \mathrm{~h}$ does not provide a reasonable fit of the clinical data.

For the late effects (see Fig. 1b), a match between clinically equivalent schedules, i.e. $70 \mathrm{~Gy}$ LDR at 50 cGy/h or 70 Gy ERT, can only be obtained at a repair half time of $1.4 \mathrm{~h}$. However, if the dose rate to deliver $70 \mathrm{~Gy}$ is $30 \mathrm{cGy} / \mathrm{h}$, a much longer repair half time of 2.3 $\mathrm{h}$ needs to be assumed to maintain a match with the 70 Gy ERT reference. The shaded area in Fig. 1b demarcates values of the intrinsic repair half time and BED values for late effects to match late normal tissue complications obtained with 64-76 Gy conventional ERT and a total dose of 65-70 Gy LDR brachytherapy prescribed at a reference dose rate between 30-50 cGy/h. In contrast to the range of repair half times for the acute effects within the shaded area of Fig. la (where a repair half time $>1.0 \mathrm{~h}$ is unlikely to occur), the value of the repair half time for the late effects has to be at least $1 \mathrm{~h}$ and can be as long as $3 \mathrm{~h}$. This is not an unexpected finding, because most of the experimental data on the kinetics of repair in late normal tissues revealed that the half times for the late effects vary between 1 and $2 \mathrm{~h}$. We will use these shaded areas as derived in Fig. 1 (boundaries of which are based on clinical experience obtained with conventional ERT and LDR brachytherapy in head and neck cancer), to serve as clinical guideline in the design of new treatment strategies for HDR and PDR bachytherapy and to check the impact of variations in the repair half time. The BED lines of 70 Gy LDR at a dose rate of $50 \mathrm{cGy} / \mathrm{h}$ and $70 \mathrm{~Gy}$ ERT serve as reference. In this way it can be easily seen that it is not realistic to assume a repair half time for the late effects of $3 \mathrm{~h}$, and to calculate subsequently an isoeffective dose for a HDR or PDR treatment schedule with 70 Gy LDR brachytherapy at $50 \mathrm{cGy} / \mathrm{h}$ as reference treatment schedule (this would imply a biologically equivalent total dose of conventional ERT of $>100 \mathrm{~Gy}$ ).

\subsection{New treatment strategies: $H D R$ and PDR brachy- therapy}

Based on practical considerations in the clinical use of brachytherapy, the following more convenient treatment schedules are chosen:

-18 fractions HDR $(=120 \mathrm{~Gy} / \mathrm{h})$ of $3.1 \mathrm{~Gy}$ for 6 con- 
secutive days, with 3 fractions per day and $6 \mathrm{~h}$ interval. No irradiation during the night.

-48 pulses of $1.4 \mathrm{~Gy}$ at a dose rate of $3 \mathrm{~Gy} / \mathrm{h}$ continuously day and night for 6 consecutive days with a pulse frequency of $3 \mathrm{~h}$, or

- 90 pulses of $0.7 \mathrm{~Gy}$ at a dose rate of $3 \mathrm{~Gy} / \mathrm{h}$ during daytime for 6 consecutive days with a pulse frequency of $1 \mathrm{~h}$. No irradiation during the night.

All these treatment schedules have the practical advantage that the patient can be disconnected from the afterloading PDR machine during the night and/or between the pulses. For reasons of comparison the optimum PDR brachytherapy irradiation scheme as proposed by Brenner and Hall [4] and Fowler [13] is also given, namely,

-140 pulses of $0.5 \mathrm{~Gy}$ continuously day and night, with a pulse repetition frequency of $1 \mathrm{~h}$ and a dose rate per pulse $1 \mathrm{~Gy} / \mathrm{h}$. In this way the basic assumption is fulfilled that the treatment time and average dose rate are kept unchanged as compared to $70 \mathrm{~Gy}$ LDR brachytherapy in $140 \mathrm{~h}$ (dose rate $=50 \mathrm{cGy} / \mathrm{h}$ ).

Fig. 2 shows the different treatment strategies schematically, keeping the overall treatment time constant $(=6$ days).

In Fig. 3 we have calculated in the same way as in Fig. 1. the BED as a function of the repair time for alternative treatment strategies of HDR and PDR brachytherapy (for the formulas used in the different situations see Appendix). To prevent a significant change in the therapeutic ratio, which would be detectable in clinical practice of brachytherapy, each BED line has to cross shaded areas in each of Fig. 3a and b. For tumor control it will be an advantage if the BED line is positioned at the top of the shaded area in Fig. $3 \mathrm{a}$, for the late reacting normal tissues at the bottom of the shaded area in Fig. 3b. For the acute as well as for the late effects the BED line of 140 pulses of $0.5 \mathrm{~Gy}$ PDR overlaps almost completely the BED line of $70 \mathrm{~Gy}$ LDR at $50 \mathrm{cGy} / \mathrm{h}$. As can hardly be noticed in this figure, but explained in detail by Fowler [16], such a PDR treatment schedule will be biologically more effective than $70 \mathrm{~Gy} \mathrm{LDR}$ at $50 \mathrm{cGy} / \mathrm{h}$ if the repair half time is $<0.5 \mathrm{~h}$. However, the impact of the (lower) dose rate per pulse and such short half times may be somewhat overemphasized. Actually, for tumor control with short half times of repair $<0.5 \mathrm{~h}$, a decrease of the dose rate per pulse will include a therapeutic disadvantage, because a decrease in BED results in lower tumor control probability. For the late effects, repair half times of $<0.5 \mathrm{~h}$ are very unlikely (see shaded area in Figs. $1 \mathrm{~b}$ and $3 \mathrm{~b})$. With the restriction of repair half times to the range of values compatible with BED values within the shaded area for the late effects, the dose rate per pulse becomes irrelevant due to the small doses per pulse. It is the dose per pulse and time intervals between the pulses which are the most important factors in sparing late normal tissues; especially for half times of $>2 \mathrm{~h}$. For these longer repair half times, the late effects are no longer maximally spared during LDR irradiation or the standard PDR treatment. In contrast, a relatively large amount of damage can be repaired during the longer time intervals between the HDR fractions or PDR pulses or during the night when no pulses are delivered.

Thus, with the uncertainties about dose and dose rate in clinical practice of brachytherapy taken into account (reflected by the shaded areas), it seems to be safe to attempt the more convenient HDR or PDR treatment schedules of $18 \times 3.1$ Gy HDR or $48 \times 1.4$ Gy PDR as an alternative to LDR brachytherapy or the standard

Continuous LDR : 70 Gy in max. 140 hours (= 6 days )

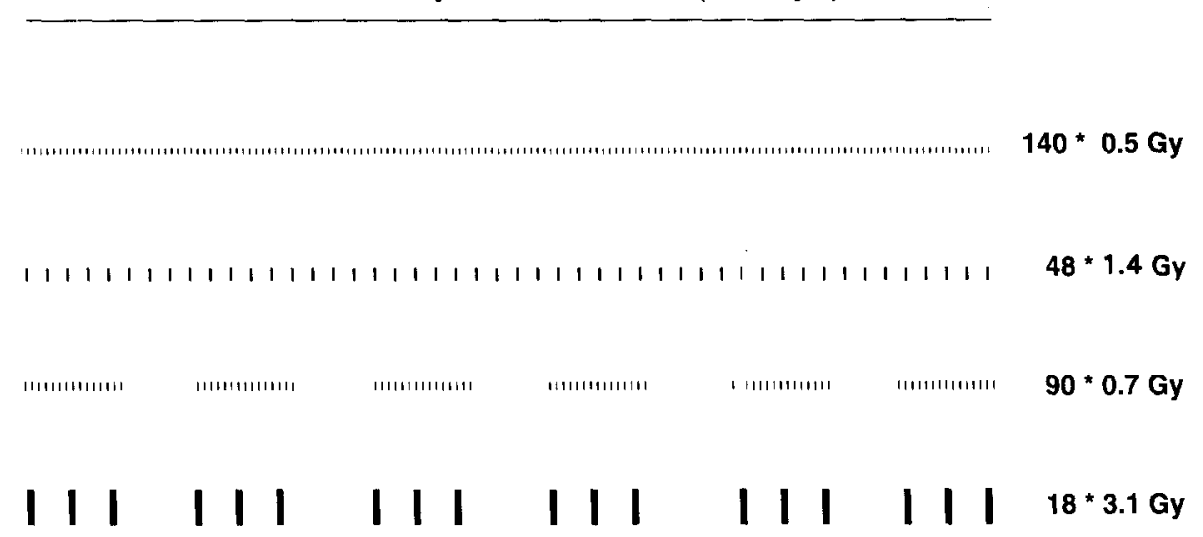

Fig. 2. Schematic representation of alternative HDR or PDR treatment strategies as compared to a standard LDR brachytherapy treatment schedule of $70 \mathrm{~Gy}$ in $140 \mathrm{~h}(=$ dose rate $50 \mathrm{cGy} / \mathrm{h})$ or the optimum PDR irradiation scheme as proposed by Brenner and Hall [4] and Fowler [13]. Overall treatment time of 6 days is kept unchanged in all treatment schedules. For further explanation see text. 


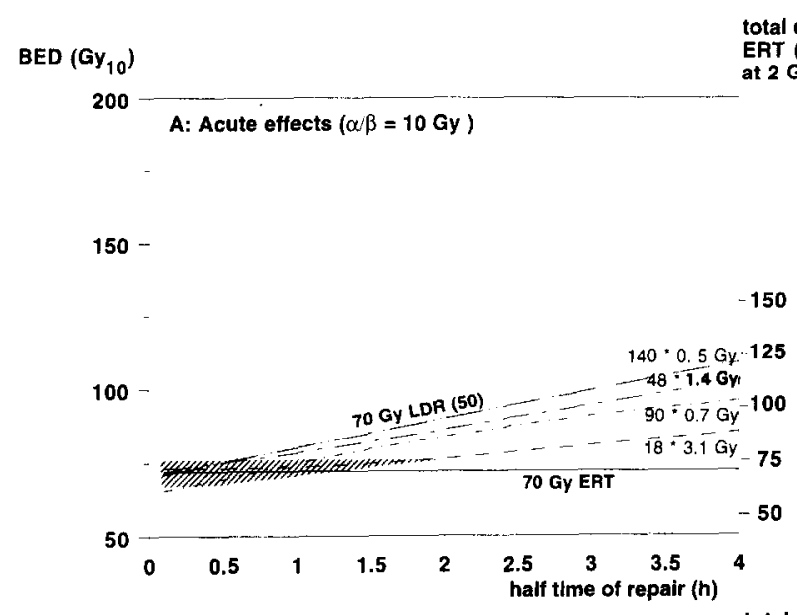
$\operatorname{BED}\left(\mathrm{Gy}_{3}\right) \quad$ ERT (Gy)

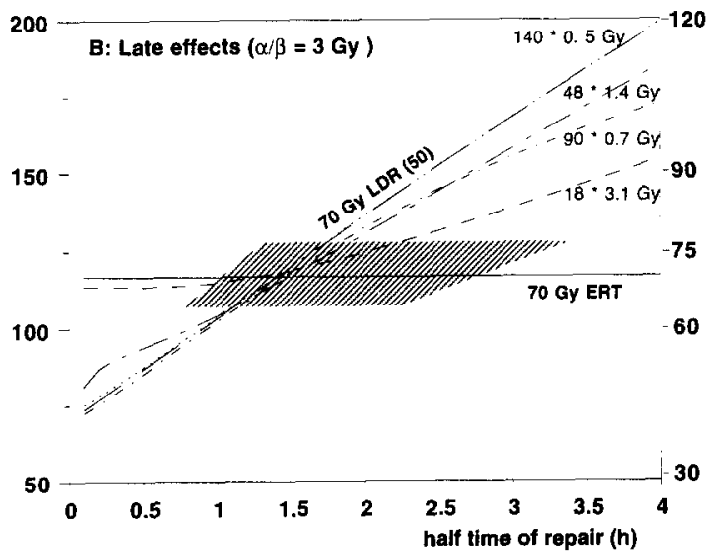

Fig. 3. Calculated BED values as a function of the repair half time for the acute effects $(A)$ and late effects (B) for the alternative HDR or PDR treatment schedules as compared with the optimum PDR brachytherapy treatment schedule as proposed by Brenner and Hall [4] and Fowler [13]. The latter is the BED line of 140 pulses of $0.5 \mathrm{~Gy}$ at a dose rate of $1 \mathrm{~Gy} / \mathrm{h}$, marked by the stippled line $(\cdots \cdots)$, which almost completely overlaps the LDR reference line of $70 \mathrm{~Gy}$ at a dose rate of $50 \mathrm{cGy} / \mathrm{h}$ (represented by the - -- marked line). The multifractionated HDR-BED line of $18 \times 3.1$ Gy HDR is marked by (-- ), the two BED lines of the PDR treatment schedules of $48 \times 1.4 \mathrm{~Gy}$ and $90 \times 0.7 \mathrm{~Gy}$ at a dose rate of $3 \mathrm{~Gy} / \mathrm{h}$ are marked by (-- ) and (----), respectively. The 70 Gy ERT reference line is the solid horizontal line $(-)$.

PDR treatment schedule. For 90 pulses of $0.7 \mathrm{~Gy}$ at a dose rate of $3 \mathrm{~Gy} / \mathrm{h}$, tumor control can be negatively affected because for a repair half time $<0.5 \mathrm{~h}$ this linc is positioned at the bottom of the shaded area of Fig. 3a. In this case 90 pulses of $0.75 \mathrm{~Gy}$ (irrespective of the dose rate per pulse) would be a better option, with slightly negative implications in sparing the late effects.

\subsection{Example of a potential pitfall of extending the BED} concept with the assumption of a longer repair half time and a $L D R$ reference scheme

To illustrate the possible risk of assumptions of the kinetics of repair in situations quite different from the one

\section{NTCP (\%)}

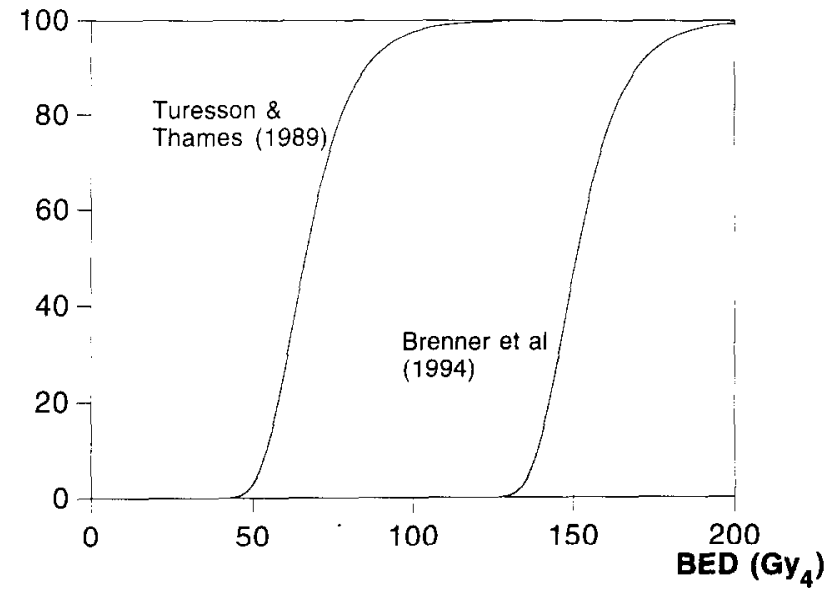

Fig. 4. Normal tissue complication probability (\%NTCP) calculated as a function of BED (in $\mathrm{Gy}_{4}$ ) for the original clinical data of Turesson and Thames [35] and for the theoretical situation with the assumptions made by Brenner et al. [6]. In the first case $\alpha / \beta=4 \mathrm{~Gy}, \alpha=0.1 \mathrm{~Gy}^{-1}$ and $k_{\mathrm{L}}=528$ (see Table $4 \mathrm{c}$ in ref [35]) is used, for the latter see text.

in which they were derived, we reanalyzed the example Brenner et al. [6] gave in their paper 'Optimizing the time course of brachytherapy and other accelerated radiotherapeutic regimes'. For illustrative purposes, the authors used late responding tissue parameters taken from the clinical data of Turesson and Thames [35], and made two main assumptions:

- a slow component of repair of $4 \mathrm{~h}$ for the late effects, without any contribution of the fast component.

- a 'generic' continuous LDR treatment of $60 \mathrm{~Gy}$ in $120 \mathrm{~h}$ produces a normal tissue complication probability of $20 \%$.

Using the formulas as described previously, it can easily be calculated that with an $\alpha / \beta$ ratio of $4 \mathrm{~Gy}$, a repair half time of $4 \mathrm{~h}$ and a total dose of $60 \mathrm{~Gy} \mathrm{LDR}$ in $120 \mathrm{~h}$, the corresponding $\mathrm{BED}_{\mathrm{LDR}}$ value is $142 \mathrm{~Gy}_{4}$. In terms of a clinical reference dose of conventional ERT at $2 \mathrm{~Gy} / \mathrm{day}$ and complete repair between the fractions, a BED $D_{\text {ERT }}$ value of $142 \mathrm{~Gy}_{4}$ would imply that a total dose of $95 \mathrm{~Gy}$ can be delivered. Obviously, such a dose would not be considered as feasible in a clinical situation. Inspecting the original clinical data of Turesson and Thames and the derived dose-response curve for teleangiectasia score $\geq 2$ at 5 years, if 5 fractions of 2 Gy/week are delivered (Fig. $3 b$ in ref [35]), a total dose of $95 \mathrm{~Gy}$ is well exceeding the $80 \mathrm{~Gy}$, which is associated with a complication probability rate of $100 \%$.

In Fig. 4 we have reconstructed the original dose response curve of Turesson and Thames [35] in terms of BED $_{\text {ERT }}$ (in $\mathrm{Gy}_{4}$ ) on the $\mathrm{X}$-axis and the probability of a teleangiectasia score $\geq 2$ at 5 years on the $Y$-axis (for the 
formulas used see the Appendix). Together with this dose-response curve based on clinical data, we constructed a complete dose-response curve for the theoretical situation with the assumptions made by Brenner et al. [6]. For this purpose we calculated the NTCP as a function of $\mathrm{BED}_{\mathrm{LDR}}$ (in $\mathrm{Gy}_{4}$ ) using a value of $2.5 \times 10^{6}$ for the parameter $K_{\mathrm{L}}$, which was calculated by Brenner et al. to be the number of target cells/colony forming units. In the ideal situation the mathematically calculated dose-response curve, based on assumed values for the different parameters characterizing the late reacting normal tissues, should overlap the original dose-response curve of Turesson and Thames, derived from actual clinical experience. However, the calculated curve for the 'generic' continuous LDR treatment schedule as assumed by Brenner et al. is shifted considerably by almost a factor two. This large difference is caused in essence by assuming a high value for the repair half time $(=4 \mathrm{~h})$ in combination with a LDR reference treatment schedule.

\section{Discussion}

Careful clinical observations will remain a prerequisite when new treatment options of HDR or PDR brachytherapy based on isoeffective dose calculations are introduced in the clinic. Especially if generalized values for the half times of repair [13] or values obtained from cells in culture [4] or hyperfractionated external beam irradiation [6] are used, it is important to realise the impact of such values for the calculation of the isoeffective dose of a reference continuous LDR treatment schedule within the conditions of incomplete repair. The potential pitfall of adopting radiobiological parameters and to apply these values in current models for the design of new treatment strategies in clinical use has been clearly shown in Fig. 4.

As a clinical guideline and to check the impact of variations of the repair half time values for both the acute and late effects, we have compared the calculated BED values in conditions of incomplete repair during continuous LDR brachytherapy with the biological effect of a clinically widely accepted reference treatment under the conditions of complete repair, i.e. a conventional ERT schedule with $2 \mathrm{~Gy}$ per fraction (see Fig. 1). Subsequently, a range of values for the repair half time of acute and late responding tissues is demarcated which reasonably can fit the clinical experience of continuous LDR brachytherapy and conventional ERT in head and neck cancer. Within the range of clinical uncertainties of dose and dose rate in clinical practice of LDR brachytherapy (reflected by the shaded areas) for the acute effects or tumor control, the best fits are obtained for repair half times of about $0.5 \mathrm{~h}$, while for the late effects the repair half times are at least $1 \mathrm{~h}$ and can be as high as $3 \mathrm{~h}$. This range of values for the repair half times for the acute and late effects is in agreement with most experimental data of the kinetics of repair studied in vitro (for review see ref. [4]) or in vivo (for review table see ref. [21]). As has become clear from Figs. 1 and 3, within these ranges of repair half times for acute and late effects, it is predominantly the late reacting normal tissues with the longer half times of repair for which problems are expected in the introduction of PDR brachytherapy as an alternative to continuous LDR brachytherapy. In this regard, the title of the paper of Fowler, 'Why shorter half times of repair lead to greater damage in pulsed brachytherapy' [16], can be misleading with regard to the late effects, because this statement is only true if one is changing from a continuous LDR treatment schedule to PDR brachytherapy within the conditions of a fixed overall treatment time and the same total dose and average dose rate. For half times $>1.5 \mathrm{~h}$, a continuous LDR treatment schedule of $70 \mathrm{~Gy}$ in $140 \mathrm{~h}$ has a relatively high BED value (compared with the biological effect of a clinical reference dose of conventional ERT with $2 \mathrm{~Gy} / \mathrm{day}$ and complete repair between the fractions) and for the late effects it is misleading to invoke biological equivalence with such a reference treatment schedule. The impact of the assumption of even higher values up to $4 \mathrm{~h}$ for the repair half times of the late effects [6] in combination with a continuous LDR reference treatment schedule is shown in Fig. 4, leading to clinically unrealistic high BED values as compared to the actual clinical experience.

For these long repair half times, the late reacting normal tissues are no longer maximally spared during a continuous LDR treatment regime of $70 \mathrm{~Gy}$ in $140 \mathrm{~h}$ as compared to other HDR and/or PDR brachytherapy treatment schedules with a fixed total dose and overall treatment time (for detailed calculations see Brenner et al., [6]). Moreover, we have shown in our calculations that, if these longer repair half times are considered for the late effects, there is no or little gain to be expected by decreasing the dose rate per pulse during PDR brachytherapy (due to the small doses per pulse), and the sparing effect for the late reacting normal tissues has to come from longer time intervals. In this scope it is not surprising that with the more convenient HDR schedule of $18 \times 3.1$ Gy or PDR schedule of $48 \times 1.4$ Gy at 3 $\mathrm{Gy} / \mathrm{h}$ the same or even better sparing effects of late normal tissues is to be expected as compared to a continuous LDR brachytherapy treatment schedule of 70 Gy in $140 \mathrm{~h}$ or the 'standard' PDR treatment schedule of $70 \mathrm{~Gy}$ with a pulse repetition frequency of $1 \mathrm{~h}$ and a dose rate per pulse of $1 \mathrm{~Gy} / \mathrm{h}$. Essential in our calculations is that total doses and average dose rates are not the same as compared with continuous LDR brachytherapy, which is different from the basic assumption of Brenner and Hall [4] and Fowler [13]. But, if theoretical models are used to predict isoeffective dose levels, why should a change in total dose and average dose rate not 
be considered? In clinical practice of external beam irradiation with accelerated and/or hyperfractionated treatment schedules, it is now generally accepted that based on isoeffective dose calculations the total dose has to be changed in dependence of the dose per fraction and the overall treatment time. Therefore, there seems to be no reason why it should not be allowed to change the total dose and average dose rate if PDR or even HDR brachytherapy treatment schedules are introduced in clinical practice as an alternative for LDR brachytherapy.

With the introduction of accelerated and/or hyperfractionated irradiation schemes in clinical practice of external beam irradiation, the interest and research in the kinetics of repair has increased. In all our theoretical calculations we assumed that the kinetics of repair is a mono-exponential function of time and dose rate independent. However, extended multifractionation studies (both clinical and experimental in vivo) revealed that the kinetics of repair might consist of a fast and a slow repair component. The analysis of acute and late human skin reactions based on a bi-exponentional model as proposed by Thames [32], showed for the acute effects a possible biphasic repair rate with a fast repair component of $0.3-0.4 \mathrm{~h}$ and a slow component of 1.1 and $1.3 \mathrm{~h}$; for the late reactions this was about $0.4 \mathrm{~h}$ and $3.5 \mathrm{~h}$, respectively [35]. A similar trend has been found for pig skin [36], spinal cord [1] and lung [37]. The proportion of the amount of damage repaired with the fast or slow component varied for the different normal tissues studied. There are no data available which indicate a biphasic pattern of the kinetics of repair during continuous LDR irradiation at a dose rate $<1 \mathrm{~Gy} / \mathrm{h}$, which is a relevant dose rate in clinical practice of LDR or PDR brachytherapy. Theoretically one could imagine that if the rate of repair indeed consists of a fast and a slow component during this (very) low dose rate irradiation, the fast component will be able to repair a relatively larger amount of damage during the protracted time at which the irradiation is delivered. The relative faster repair kinetics after continuous low dose rate irradiation found in vitro [30] or in vivo [11] may sustain this theoretical consideration, but at least indicates that one has to be careful with a direct translation of either one or two components of repair found in the ERT experimental or clinical situation to the daily practice of brachytherapy. More experimental data in well characterized and clinically relevant animal models are needed to test the applicability of a mono- or bi-exponential IR model during continuous irradiations, especially under the brachytherapy conditions of the rapid dose fall off.

Finally, clinical considerations about consequences of practical use and patient convenience are equally important. In this regard, we have shown that, with constraints in the use of repair half times in mathematical modelling of clinical HDR and PDR treatment schedules, it will probably be difficult to detect any sig- nificant difference in clinical practice between the more convenient HDR or PDR treatment schedules of $18 \mathrm{x}$ 3.1 Gy HDR or $48 \times 1.4 \mathrm{~Gy} \mathrm{PDR}$ at $3 \mathrm{~Gy} / \mathrm{h}$ as compared to LDR brachytherapy or the PDR treatment schedule as proposed by Brenner and Hall [4] and Fowler [13]. However, before these alternative treatment options are introduced in the clinic, a thorough experimental investigation and a detailed knowledge of the radiobiological repair factors for specific tissues and endpoints is warranted.

\section{Appendix}

\section{Isoeffect models based on the BED concept}

\section{Infinite dose-rate, complete repair}

$B E D=(n d) \cdot\left(1+\frac{d}{\alpha / \beta}\right)-\frac{\gamma T}{\alpha}$

BED $=$ biological equivalent dose,

$n \quad=$ the number of fractions,

$d \quad=$ the dose per fraction,

$\alpha / \beta=$ the repair constant $(=$ fractionation sensitivity) of the tissue involved,

$n d=$ the dosage factor $=$ the total dose,

$T \quad=$ the time during which proliferation occurs after any initial time lag, $T=T_{\text {overall }}-T_{\mathrm{K}}$, and $T_{\mathrm{K}}$ is the kickoff time,

$\gamma=$ the assumed constant rate at which proliferation occurs, $\gamma=\ln 2 / T_{\text {pot }}$ and $T_{\text {pot }}$ is the average doubling time of the proliferating cells.

2. Infinite dose-rate, incomplete repair

$\left.\left.B E D=(n d) \cdot\left(1+\frac{d}{\alpha / \beta}\right)\left[1+h_{\mathrm{n}} \theta\right)\right]\right)-\frac{\gamma T}{\alpha}$

$h_{n}(\theta)$ represents the amount of unrepaired damage, which depends on:

$n=$ the number of fractions,

$T_{1 / 2}=$ the assumed constant value of the half time at which repair proceeds,

$t \quad=$ the time between subsequent fractions, during which repair can occur.

Special cases for $h_{\mathrm{n}}(\theta)$ :

- when $n$ equi-spaced fractions are given $h_{\mathrm{n}}$ is presented by:

$h_{n}(\theta)=\left(\frac{2}{n}\right) \cdot\left(\frac{\theta}{1-\theta}\right) \cdot\left(n-\frac{1-\theta^{n}}{1-\theta}\right)$

$\theta \quad=\mathrm{e}^{-\mu t}, T_{1 / 2}=\ln (2) / \mu$

$\boldsymbol{\theta}=$ the monoexponential repair function, depending on $T_{1 / 2}$,

$\mu \quad=$ the parameter for the kinetics of repair. 
- when 2 fractions are given during day-time, with incomplete repair overnight, $h_{\mathrm{n}}(\theta)$ is presented by:

$$
\begin{gathered}
h_{n}\left(\theta_{1,2}\right)=\theta_{1}+\left(\frac{2}{n}\right) \cdot \theta_{2} \cdot \frac{\left(1+\theta_{1}\right)^{2}}{1-\theta_{1} \cdot \theta_{2}} . \\
{\left[\frac{n}{2}-\frac{1-\left(\theta_{1} \theta_{2}\right)^{n / 2}}{1-\theta_{1} \theta_{2}}\right]}
\end{gathered}
$$

$\theta_{1}=\mathrm{e}^{-\mu t}, \theta_{2}=\mathrm{e}^{-\mu[24-t]}$

$t=$ the time between fractions during day-time,

$24-t=$ the repair time overnight.

- when 3 fractions are given during day-time, with incomplete repair overnight, $h_{n}(\theta)$ is presented by:

$$
\begin{gathered}
h_{n}\left(\theta_{1}, \theta_{2}, \theta_{3}\right)=\left(\frac{2}{n}\right) \cdot\left[\frac{n}{3} \cdot\left(\theta_{1}+\theta_{2}+\theta_{1} \theta_{2}\right)\right. \\
\left.+\frac{K}{1-\theta_{1} \theta_{2} \theta_{3}} \cdot \frac{1-\theta_{1} \theta_{2} \theta_{3}{ }^{n / 3}}{1-\theta_{1} \theta_{2} \theta_{3}}\right]
\end{gathered}
$$

$K=\theta_{3} \cdot\left[\theta_{1} \theta_{2}+\left(1+\theta_{1} \theta_{2}\right)\left(1+\theta_{1}\right)\left(1+\theta_{2}\right)\right]$

$$
\theta_{\mathrm{i}}=\mathrm{e}^{-\mu r_{\mathrm{i}}}
$$

$t 1=$ the time between fraction 1 and 2 ,

$t 2=$ the time between fraction 2 and 3 ,

$t 3=$ the repair time overnight.

- for a real scheme, with fraction $i, t_{\mathrm{i}} \mathrm{h}$ after fraction $i-1$ and dose $d_{i}$, a series for BED can be calculated as follows:

$\operatorname{BED}_{\mathrm{i}}=d_{\mathrm{i}} \cdot\left(1+\frac{d_{\mathrm{i}}}{\alpha / \beta}\left[1+2 \cdot F_{\mathrm{i}}\right]\right)-\frac{\gamma T}{\alpha}$

$F_{\mathrm{i}}=\theta_{\mathrm{i}} \cdot\left(1+F_{\mathrm{i}-1}\right), F_{1}=0$

$\theta_{\mathrm{i}}=\mathrm{e}^{-\mu t}$

For the total effect:

$\mathrm{BED}=\sum_{i=1}^{n} \mathrm{BED}_{\mathrm{i}}$

\section{Low dose-rate continuous irradiation}

$\mathrm{BED}=d \cdot\left(1+\frac{d}{\alpha / \beta}[g(\mu t)]\right)$

$d=$ dose $=v t$, where $v=$ dose rate; $t=$ irradiation time.

$g(\mu t)=2 \cdot\left(\mu \mathrm{t}-1+\mathrm{e}^{-\mu t}\right) /(\mu t)^{2}$

\section{Fractionated low dose-rate irradiation.}

- when the fractionated doses are equally spaced, BED is represented by:

$$
\begin{aligned}
& \operatorname{BED}=n \cdot d_{n} \cdot\left(1+\frac{d_{n}}{\alpha / \beta} \cdot\left[g\left(\mu t_{n}\right)\right.\right. \\
& \left.\left.+g^{\prime}\left(\mu t_{n}\right) \cdot h_{n}(\theta)\right]\right) \\
& g^{\prime}\left(\mu t_{n}\right)=\frac{\mathrm{e}^{\mu t_{n}}-2+\mathrm{e}^{-\mu t_{n}}}{\left(\mu t_{n}\right)^{2}} \\
& \Theta=\mathrm{e}^{-\mu\left(t_{n}+\delta t_{n}\right)}
\end{aligned}
$$

$d_{n}=v t_{n}$, where $t_{n}=$ exposure time per fraction; $\delta t_{\mathrm{n}}=$ time between fractions.

- for a real scheme, fraction $i$, with dose $d_{\mathrm{i}}$ given after $\delta t_{\mathrm{i}} \mathrm{h}$ after fraction $i-1$, a series for BED can be calculated as follows:

$\mathrm{BED}_{\mathrm{i}}=d_{\mathrm{i}} \cdot\left(1+\frac{d_{\mathrm{i}}}{\alpha / \beta} \cdot\left[g\left(\mu t_{\mathrm{i}}\right)+g^{\prime}\left(\mu t_{\mathrm{i}}\right) \cdot 2 \cdot F_{\mathrm{i}}\right]\right)$

$F_{\mathrm{i}}=\theta_{\mathrm{i}} \cdot\left(1+F_{i-1}\right), F_{1}=0$

$\theta_{\mathrm{i}}=\mathrm{e}^{-\mu\left(t_{\mathrm{i}}+\delta t_{\mathrm{i}}\right)}$

For the total effect:

$\mathrm{BED}=\Sigma_{\mathrm{i}=1}^{n} \mathrm{BED}_{\mathrm{i}}$

\section{Normal tissue complication probability}

- from the BED calculated for a treatment schedule, the NTCP follows from:

$\mathrm{NTCP}=\exp \left[-k_{\mathrm{L}} \cdot \exp ^{(-\alpha \cdot \mathrm{BED})}\right]$

NTCP = normal tissue complication probability,

$k_{\mathrm{L}} \quad=$ the number of tissue-rescuing units (TRUs) initially at risk for a specific late effect.

\section{Acknowledgements}

This study was supported by grant NUKC 94-725, provided by the Dutch Cancer Society. The authors wish to thank Dr. K.K. Ang for critical reading of the manuscript and helpful comments.

\section{References}

[1] Ang, K.K., Guttenberger, R., Thames, H.D., Stephens, L.C., Smith, C.D. and Feng, Y. Impact of spinal cord repair kinetics on the practice of altered fractionation schedules. Radiother. Oncol. 25: 287-294, 1992. 
[2] Barendsen, G.W. Dose fractionation, dose rate and iso-effect relationships for normal tissue responses. Int. J. Radiat. Oncol. Biol. Phys. 8: 1981-1997, 1982.

[3] Benk, V., Mazeron, J.J., Grimard, L., Crook, J., Haddad, E., Piedbois, P., Calitchi, E., Raynal, M., Martin, M., Le Bourgeois, J.P. and Pierquin, B. Comparison of curietherapie versus external irradiation combined with curietherapie in Stage II squamous cell carcinomas of the mobile tongue. Radiother. Oncol. 18: 339-447, 1990.

[4] Brenner, D.J. and Hall, E.J. Conditions for the equivalence of continuous to pulsed low dose rate brachytherapy. Int. J. Radiat. Oncol. Biol. Phys. 20: 181-190, 1991.

[5] Brenner, D.J. and Hall, E.J. Fractionated high dose rate versus low dose rate regimens for intracavitary brachytherapy of the cervix. I. General considerations based on radiobiology. Br. J. Radiol. 64: 133-141, 1991.

[6] Brenner, D.J., Hall, E.J., Huang, Y. and Sachs, R.K. Optimizing the time course of brachytherapy and other accelerated radiotherapeutic protocols. Int. J. Radiat. Oncol. Biol. Phys. 29: 893-901, 1994.

[7] Chu, A. and Fletcher, G.H. Incidence and causes of failures to control by irradiation the primary lesions in squamous cell carcinomas of the anterior two-thirds of the tongue and floor of mouth. Am. J. Roentgenol. 117: 502-508, 1973.

[8] Dale, R.G. The application of the linear-quadratic dose-effect equation to fractionated and protracted radiotherapy. Br. J. Radiol. 58: 515-528, 1985.

[9] Dale, R.G. The application of the linear-quadratic model to fractionated radiotherapy when there is incomplete normal tissue recovery between fractions, and possible implications for treatments involving multiple fractions per day. Br. J. Radiol. 59: 919-927, 1986.

[10] Dale, R.G. Time dependent tumour repopulation factors in linear quadratic equations - implications for treatment strategies. Radiother. Oncol. 15: 371-382, 1989.

[11] Dale, R.G., Huzkowsky, J. and Trott, K.R. Possible dose rate dependence of recovery kinetics as deduced from a preliminary analysis of the effects of fractionated irradiations at varying dose rates. Br. J. Radiol. 61: 153-157, 1988.

[12] Dutreix, A. and Marinello, G. The Paris system. In: Modern Brachytherapy, pp. 25-42. Editors: B. Pierquin, J.F. Wilson and D. Chassagne. Masson Publishing USA, New York, 1987.

[13] Fowler, J. and Mount, M. Pulsed brachytherapy: the conditions for no significant loss of therapeutic ratio compared with traditional low dose rate brachytherapy. Int. J. Radiat. Oncol. Biol. Phys. 23: 661-669, 1992.

[14] Fowler, J.F. How worthwhile are short schedules in radiotherapy?: A series of exploratory calculations. Radiother. Oncol. 18: 165-181, 1990.

[15] Fowler, J.F. The linear-quadratic formula and progress in fractionated radiotherapy. Br. J. Radiol. 62: 679-694, 1989.

[16] Fowler, J.F. Why shorter half-times of repair lead to greater damage in pulsed brachytherapy. Int. J. Radiat. Oncol. Biol. Phys. 26: 353-356, 1993.

[17] Fowler, J.J. Dose rate effects in normal tissues. In: Brachytherapy 2, pp. 26-40. Editor: R.F. Mould. Nucletron, Leersum, 1989.

[18] Fu, K.F., Ray, J.W., Chan, E.K. and Phillips, T.L. External and interstitial radiation therapy of carcinoma of the oral tongue. A review of 32 years' experience. Am. J. Radiol. 126: 107-115, 1976.

[19] Guttenberger, R., Thames, H.D. and Ang, K.K. Is the experience with CHART compatible with experimental data? Radiother. Oncol. 25: 280-286, 1992.

[20] Hendry, J.H. Treatment acceleration in radiotherapy: the relative time factors and dose-response slopes for tumours and normal tissues. Radiother. Oncol. 25: 308-312, 1992.
[21] Joiner, M.C. The linear-quadratic approach to fractionation. In: Basic Clinical Radiobiology for Radiation Oncologists, pp. 55-64. Editor: G.G. Steel. Edward Arnold, London, 1993.

[22] Mazeron, J.J., Crook, J.M., Marinello, G., Walop, W. and Pierquin, B. Prognostic factors of local outcome for T1, T2 carcinomas of oral tongue treated by Iridium 192 implantation. Int. J. Radiat. Oncol. Biol. Phys. 19: 281-285, 1990.

[23] Mazeron, J.J., Simon, J.M., Le Pechoux, C., Crook, J., Grimard, L., Piedbois, P., Le Bourgeois, J.P. and Pierquin, B. Effect of dose rate on local control and complications in definitive irradiation of $T_{1-2}$ squamous cell carcinomas of mobile tongue and floor of mouth with interstitial iridium-192. Radiother. Oncol. 21: 39-47, 1991.

[24] Mendenhall, W.M., Parsons, J.T., Stringer, S.P., Cassisi, N.J. and Million, R.R. $T_{2}$ oral tongue carcinoma treated with radiotherapy; analysis of local control and complications. Radiother. Oncol. 16: 275-281, 1989.

[25] Meredith, W.J. Radium Dosage. The Manchester system. Williams and Wilkins, Baltimore, 1949.

[26] Nilsson, P., Thames, H.D. and Joiner, M.C. A generalized formulation of the 'incomplete-repair' model for cell survival and tissue response to fractionated low dose-rate irradiation. Int. J. Radiat. Biol. 57: 127-142, 1990.

[27] Pierquin, B., Mazeron, J.J., Grimard, L. and Marinello, G. Normal tissue tolerance in brachytherapy. In: Radiation Tolerance of Normal Tissues, Front. Radiat. Ther. Oncol., Vol. 23, pp. 194-201. Editors: J.M. Vaeth and J.L. Meyer. Karger, Basel, 1989.

[28] Roberts, S.A. and Hendry, J.H. The delay before onset of accelerated tumor cell repopulation during radiotherapy: a direct maximum-likelyhood analysis of worldwide tumour control data. Radiother. Oncol. 29: 69-74, 1993.

[29] Steel, G.G., Deacon, J.M., Duchesne, G.M., Horwich, A., Kelland, L.R. and Peacock, J.H. The dose-rate effect in human tumour cells. Radiother. Oncol. 9: 299-310, 1987.

[30] Taylor, J.M.G. and Withers, H.R. Dose-time factors in head and neck data. Radiother. Oncol. 25: 313-315.

[31] Thames, H.D. An 'incomplete-repair' model for survival after fractionated and continuous irradiation. Int. J. Radiat. Biol. 47: 319-339, 1985.

[32] Thames, H.D. Repair kinetics in tissues: alternative models. Radiother. Oncol. 14: 321-327, 1989.

[33] Thames, H.D., Bentzen, S.M., Turesson, I., Overgaard, M. and Van den Bogaert, W. Time dose factors in radiotherapy: a review of the human data. Radiother. Oncol. 19: 219-235, 1990.

[34] Travis, E.L. and Tucker, S.L. Isoeffect models and fractionated radiation therapy. Int. J. Radiat. Oncol. Biol. Phys. 13: 283-287, 1987.

[35] Turesson, I. and Thames, H.D. Repair capacity and kinetics of human skin during fractionated radiotherapy: erythema, desquamation, and telangiectasia after 3 and 5 year's follow-up. Radiother. Oncol. 15: 169-188, 1989

[36] van den Aardweg, G.J.M.J. and Hopewell, J.W. The kinetics of repair for sublethal radiation-induced damage in the pig epidermis: an interpretation based on a fast and a slow component of repair. Radiother. Oncol. 23: 94-104, 1992.

[37] van Rongen, E., Thames, H.D. and Travis, E.L. Recovery from radiation damage in mouse lung: interpretations in terms of two rates of repair. Radiat. Res. 133: 225-233, 1993.

[38] Wang, C.C., Doppke, K.P. and Biggs, P.J. Intra-oral cone radiation therapy for selected carcinomas of the oral cavity. Int. J. Radiat. Oncol. Biol. Phys. 9: 1185-1189, 1983.

[39] Withers, H.R., Taylor, J.M.G. and Maciejewski. The hazard of accelerated tumor clonogen repopulation during radiotherapy. Acta Oncol. 27: 131-146, 1988. 